\title{
Foliations with non-compact leaves on surfaces
}

\author{
Sergiy Maksymenko Eugene Polulyakh
}

\begin{abstract}
The paper studies non-compact surfaces obtained by gluing strips $\mathbb{R} \times(-1,1)$ with at most countably many boundary intervals along some these intervals. Every such strip possesses a foliation by parallel lines, which gives a foliation on the resulting surface. It is proved that the identity path component of the group of homeomorphisms of that foliation is contractible.
\end{abstract}

Keywords harmonic function, foliation, homotopy type

UDC 515.1

\section{Introduction}

The qualitative part of one complex variable functions theory concerns with topological classification of analytical and pseudoharmonic functions as well as with foliations of their level-sets. Such kind of problems was considered by S. Stoilov [1] and G. T. Whyburn [2] who introduced notions of internal and light open maps (respectively) which reflect certain essential topological features of analytical mappings. At the same time foliations by level sets of harmonic function on the plane were studied by W. Kaplan [3].

We will say that a continuous function $f: Z \rightarrow \mathbb{R}$ agrees with a 1-dimensional foliation $\mathcal{F}$ on $Z$ if

- each leaf of $\mathcal{F}$ is a connected component of some level-set $f^{-1}(c), c \in \mathbb{R}$;

- for each $z \in \mathbb{R}^{2}$ there are local coordinates $(u, v)$ in which $z=(0,0)$ and $f(u, v)=u+$ const. 
Suppose $\mathcal{F}$ is a one-dimensional foliation on $\mathbb{R}^{2}$ with all leaves non-compact. W. Kaplan [3], [4] extending old result by E. Kamke [5] proved that then there exists a continuous function $f: \mathbb{R}^{2} \rightarrow \mathbb{R}$ which agrees with $\mathcal{F}$. Moreover, one can find at most countable covering $\left\{S_{\alpha}\right\}_{\alpha \in \mathbf{A}}$ of $\mathbb{R}^{2}$ such that

(1) each $S_{\alpha}$ consists of entire leaves of $\mathcal{F}$;

(2) the foliation on $S_{\alpha}$ is equivalent to the foliation on the plane $\mathbb{R}^{2}$ or on the half-plane by parallel lines.

In other words, $\mathbb{R}^{2}$ is glued of countably many strips along open boundary intervals, see Figure 1.1.
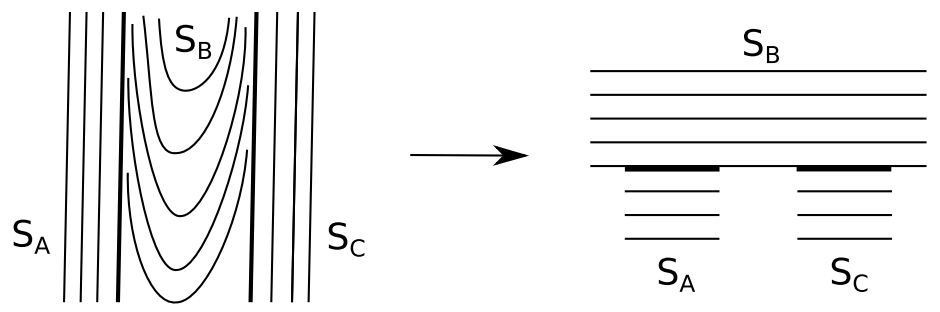

Fig. 1.1

In [6] W. Kaplan also shown that there exists a homeomorphism $h: \mathbb{R}^{2} \rightarrow \mathbb{R}^{2}$ such that the function $f \circ h$ is harmonic. This result was further extended to foliations with singularities W. Boothby [7], [8], M. Morse and J. Jenkins [9], M. Morse [10]. See also [9], [11], [12], [13], [14], [15]

In the last twenty years the interest to the topological classification of functions on surfaces arises due to a progress in the theory of Hamiltonial dynamical systems of small degrees of freedom, see e.g. A. Fomenko and A. Bolsinov [16], A. Oshemkov [17], V. Sharko [18], [19], E. Polulyakh and I. Yurchuk [20], E. Polulyakh [21].

In the present paper we will study homotopical properties of foliations having properties (1) and (2) above on arbitrary open surfaces $Z$. Thus such a surface is obtained from a family of strips $\left\{S_{\alpha}\right\}_{\alpha \in \mathbf{A}}$ glued along some boundary intervals, and therefore such surfaces will be called stripped. Every strip admits a natural foliation by parallel lines which gives a foliation $\mathcal{F}_{Z}$ on $Z$ with all leaves noncompact. We will call this foliation canonical. Let $\mathcal{H}\left(\mathcal{F}_{Z}\right)$ be the group of all homeomorphisms of $Z$ which maps leaves of $\mathcal{F}_{Z}$ onto leaves of $\mathcal{F}_{Z}$, and $\mathcal{H}_{0}\left(\mathcal{F}_{Z}\right)$ be the identity path component of $\mathcal{H}\left(\mathcal{F}_{Z}\right)$ with respect to the open compact topology. We will prove (Theorem 4.4$)$ that $\mathcal{H}_{0}\left(\mathcal{F}_{Z}\right)$ is contractible. Hence the 
homotopy type of $\mathcal{H}\left(\mathcal{F}_{Z}\right)$ reduces to the computation of the homeotopy group (or mapping class group) $\pi_{0} \mathcal{H}\left(\mathcal{F}_{Z}\right)=\mathcal{H}\left(\mathcal{F}_{Z}\right) / \mathcal{H}_{0}\left(\mathcal{F}_{Z}\right)$ of the foliation $\mathcal{F}_{Z}$. As an example we characterize stripped surfaces consisting of one strip (Theorem 3.7).

\section{Stripped surfaces}

Definition 2.1 A subset $S \subset \mathbb{R} \times[-1,1]$ will be called a model strip if

(1) $\mathbb{R} \times(-1,1) \subset S$,

(2) the intersection $S \cap(\mathbb{R} \times\{-1,1\})$ is a (possibly empty) union of open finite intervals with mutually disjoint closures.

For example, $\mathbb{R} \times(-1,1)$ is a model strip, while $\mathbb{R} \times[-1,1], \mathbb{R} \times(-1,1]$, $\mathbb{R} \times[-1,1)$ are not.

For a model strip $S$ we will use the following notation:

$\partial_{-} S:=S \cap \mathbb{R} \times\{-1\}, \quad \partial_{+} S:=S \cap \mathbb{R} \times\{1\}, \quad \partial S:=\partial_{-} S \cup \partial_{+} S$.

Connected components of $\partial_{-} S\left(\operatorname{resp} . \partial_{+} S\right)$ will be called lower (resp. upper) boundary intervals.

Definition 2.2 A stripped surface is the quotient space

$$
Z=\bigsqcup_{\alpha \in \mathbf{A}} S_{\alpha} /\left\{Y_{\beta} \stackrel{\phi_{\beta}}{\sim} X_{\beta}\right\}_{\beta \in \mathbf{B}}
$$

where

(a) $\bigsqcup_{\alpha \in \mathbf{A}} S_{\alpha}$ is a disjoint union of model strips;

(b) $\left\{X_{\beta}, Y_{\beta}\right\}_{\beta \in \mathbf{B}} \subset \bigcup_{\alpha \in \mathbf{A}} \partial S_{\alpha}$ is a family of pairs of boundary intervals such that $X_{\beta} \neq Y_{\beta}, Y_{\beta} \neq Y_{\beta^{\prime}}$ and $X_{\beta} \neq X_{\beta^{\prime}}$ for $\beta \neq \beta^{\prime} \in \mathbf{B}$;

(c) $\phi_{\beta}: Y_{\beta} \rightarrow X_{\beta}, \beta \in \mathbf{B}$, is an affine homeomorphism preserving or reversing orientations.

Thus a stripped surface is a surface obtained from a family model strips by identifying some pairs of boundary intervals via affine homeomorphisms. It is allowed that two strips are glued along more than one pair of boundary components. One may also glue together intervals belonging to the boundary of same strip $S$, and even to the same lower or upped part of $\partial S$. 
Remark 2.3 Notice that if $a<b$ and $c<d$, then there are exactly two affine homeomorphisms $\phi^{+}, \phi^{-}:(a, b) \rightarrow(c, d)$ such that $\phi^{+}$preserves orientation and $\phi^{-}$reverses it. Namely,

$$
\phi^{+}(t)=\frac{d-c}{b-a}(t-a)+c, \quad \phi^{-}(t)=\frac{c-d}{b-a}(t-a)+d,
$$

for $t \in(a, b)$.

Remark 2.4 The assumption that the gluing maps are affine is technical and not crucial, however it will be essentially used in the proof of Lemma 4.3.

Let $Z$ be a stripped surface, defined by (2.1), and

$$
q: \bigsqcup_{\alpha \in \mathbf{A}} S_{\alpha} \longrightarrow Z
$$

be the quotient map. Then $Z$ is a non-compact two-dimensional manifold which can be nonconnected, non-orientable, and have boundary. Every connected component of $\partial Z$ is an interval. Also notice that a subset $U \subset Z$ is open if and only if $q^{-1}(U)$ is open in $\bigsqcup_{\alpha \in \mathbf{A}} S_{\alpha}$.

For each $\alpha \in \mathbf{A}$ let

$$
\xi_{\alpha}: S_{\alpha} \hookrightarrow \bigsqcup_{\alpha \in \mathbf{A}} S_{\alpha} \stackrel{q}{\longrightarrow} Z
$$

be the composition of the inclusion of $S_{\alpha}$ into $\bigsqcup_{\alpha \in \mathbf{A}} S_{\alpha}$ with the quotient map $q$. We will call $\xi_{\alpha}$ a chart map corresponding to $S_{\alpha}$.

It will also be convenient to use the following notation:

$$
\widehat{S}_{\alpha}=\xi_{\alpha}\left(S_{\alpha}\right), \quad \partial_{-} \widehat{S}_{\alpha}=\xi_{\alpha}\left(\partial_{-} S_{\alpha}\right), \quad \partial_{+} \widehat{S}_{\alpha}=\xi_{\alpha}\left(\partial_{+} S_{\alpha}\right), \quad \partial \widehat{S}_{\alpha}=\xi_{\alpha}\left(\partial S_{\alpha}\right)
$$

In particular, the image $\widehat{S}_{\alpha}=\xi_{\alpha}\left(S_{\alpha}\right)$ will be called a strip of $Z$. Notice that if $\xi_{\alpha}$ is not an embedding, then $\partial_{-} \widehat{S}_{\alpha}$ and $\partial_{+} \widehat{S}_{\alpha}$ may intersect.

On the other hand, the assumptions (b) and (c) guarantee that both restrictions $\left.\xi_{\alpha}\right|_{\partial_{-} S_{\alpha}}$ and $\left.\xi_{\alpha}\right|_{\partial_{+} S_{\alpha}}$ are injective.

\section{Canonical foliation on a stripped surface}

Notice that each model strip $S$ admits a one-dimensional foliation $\mathcal{F}_{S}$ whose leaves are connected components of $\partial S$ and sets $\mathbb{R} \times\{t\}, t \in(-1,1)$.

More generally, let $Z$ be a stripped surface. Since its strips are glued by homeomorphisms of leaves, the foliations on strips of $Z$ yield a foliation $\mathcal{F}_{Z}$ on $Z$. We will call this foliation canonical. 
Let $\Gamma(\mathcal{F})=Z / \mathcal{F}$ be the space of leaves endowed with the corresponding quotient topology, and $p: Z \rightarrow \Gamma(\mathcal{F})$ be the quotient map. Then by definition a subset $V \subset \Gamma(Z)$ is open if and only if its inverse image $p^{-1}(V)$ is open in $Z$. Thus open subsets of $\Gamma(\mathcal{F})$ can be regarded as open saturated subsets of $Z$.

Lemma 3.1 $\Gamma(\mathcal{F})$ is a $T_{1}$-space.

Proof We should prove that every one-point set $\{x\} \subset \Gamma(\mathcal{F})$ is closed in $\Gamma(\mathcal{F})$, i.e. that each leaf $\omega$ of $\mathcal{F}$ is closed in $Z$. Since $Z=\bigsqcup_{\alpha \in \mathbf{A}} S_{\alpha} /\left\{Y_{\beta} \stackrel{\phi_{\beta}}{\sim} X_{\beta}\right\}_{\beta \in \mathbf{B}}$, see (2.1), we should check that every leaf in a model strip is closed. But the latter is evident for leaves belonging to interiors of strips $\operatorname{Int} S_{\alpha}=\mathbb{R} \times(-1,1)$ and follows from (2) of Definition 2.1 for leaves belonging to $\partial S_{\alpha}=S_{\alpha} \backslash \operatorname{Int} S_{\alpha}$.

A homeomorphism $h: Z \rightarrow Z^{\prime}$ between stripped surfaces will be called an $\mathcal{F}$-homeomorphism whenever it maps leaves of $\mathcal{F}_{Z}$ onto leaves of $\mathcal{F}_{Z^{\prime}}$.

Evidently, for each leaf $\omega$ we have exactly one of the following possibilities.

(a) $\omega$ belongs to $\widehat{S}_{\gamma} \backslash \partial \widehat{S}_{\gamma}$ for some $\gamma \in \mathbf{A}$; in this case $\omega$ will be called internal.

(b) $\omega \subset \partial \widehat{S}_{\gamma} \subset \partial Z$ for some $\gamma \in \mathbf{A}$; in this case $\omega$ will be called a boundary leaf.

(c) $\omega \subset \partial_{\varepsilon} \widehat{S}_{\gamma} \cap \partial_{\varepsilon^{\prime}} \widehat{S}_{\gamma^{\prime}}$ for some $\gamma, \gamma^{\prime} \in \mathbf{A}$ and $\varepsilon, \varepsilon^{\prime} \in\{ \pm\}$. Then

$$
\omega=\xi_{\gamma}\left(X_{\beta}\right)=\xi_{\gamma^{\prime}}\left(Y_{\beta}\right)
$$

for some $\beta \in \mathbf{B}$. This situation splits into the following three cases:

(c1) $\gamma=\gamma^{\prime}, X=\partial_{\varepsilon} S_{\gamma}$, and $Y=\partial_{\varepsilon^{\prime}} S_{\gamma}$; so $\omega=\partial_{-} \widehat{S}_{\gamma}=\partial_{+} \widehat{S}_{\gamma}=\partial \widehat{S}_{\gamma}$;

(c2) $\gamma \neq \gamma^{\prime}, X=\partial_{\varepsilon} S_{\gamma}$, and $Y=\partial_{\varepsilon^{\prime}} S_{\gamma}$, so $\omega=\partial_{\varepsilon} \widehat{S}_{\gamma}=\partial_{\varepsilon^{\prime}} \widehat{S}_{\gamma^{\prime}}$;

(c3) $X \neq \partial_{\varepsilon} S_{\gamma}$ or $Y \neq \partial_{\varepsilon^{\prime}} S_{\gamma}$, in this case $\omega$ will be called special.

Our aim is to reduce the situation to the case when there is no leaves of types (c1) and (c2). For this we need the following lemma.

Lemma 3.2 Let $S=\mathbb{R} \times(-1,1)$ and $T=S \backslash((-\infty,-1] \cup[1,+\infty)) \times 0$. Then there exists a homeomorphism $h: S \rightarrow T$ fixed outside $\mathbb{R} \times\left(-\frac{1}{2}, \frac{1}{2}\right)$ and preserving horizontal lines, that is $h(x, y)=(\alpha(x, y), y)$ for some continuous function $\alpha$, see Figure 3.1.

Proof Let $\sigma: \mathbb{R} \rightarrow(-1,1)$ be a $C^{\infty}$-diffeomorphism given by $\sigma(t)=\frac{t}{t^{2}+1}$. Evidently, $\sigma^{\prime}(t)=\frac{1-t^{2}}{\left(1+t^{2}\right)^{2}}$. Define $h: S \rightarrow T$ by the formula: 


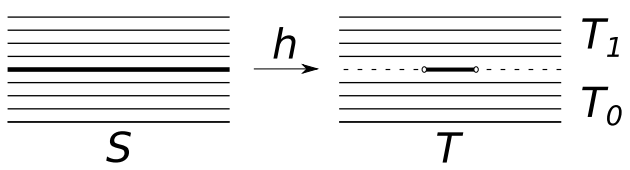

Fig. 3.1

$$
h(x, y)= \begin{cases}(\sigma(x), 0), & y=0, \\ \left(\sigma^{\prime}\left(-\frac{1}{|y|}\right) \cdot\left(x+\frac{1}{|y|}\right)+\sigma\left(-\frac{1}{|y|}\right), y\right), & y \neq 0, x \in\left(-\infty,-\frac{1}{|y|}\right] \\ (\sigma(x), y), & y \neq 0, x \in\left[-\frac{1}{|y|}, \frac{1}{|y|}\right] \\ \left(\sigma^{\prime}\left(\frac{1}{|y|}\right) \cdot\left(x-\frac{1}{|y|}\right)+\sigma\left(\frac{1}{|y|}\right), y\right), & y \neq 0, x \in\left(\frac{1}{|y|},+\infty\right) .\end{cases}
$$

The verification that $h$ is indeed a homeomorphism having the corresponding properties we leave for the reader.

Corollary 3.3 Let $S=\mathbb{R}^{2}$ and $T=\mathbb{R}^{2} \backslash((-\infty,-1] \cup[1,+\infty)) \times\{2 n\}_{n \in \mathbb{Z}}$. Then there exists a homeomorphism $h: S \rightarrow T$ fixed outside $\mathbb{R} \times \cup_{n \in \mathbb{Z}}\left(2 n-\frac{1}{2}, 2 n+\frac{1}{2}\right)$ and preserving horizontal lines, that is $h(x, y)=(\alpha(x, y), y)$ for some continuous function $\alpha$, see Figure 3.2.

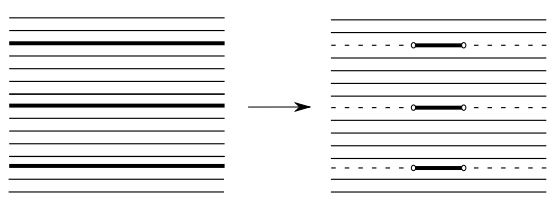

Fig. 3.2

Remark 3.4 Notice that $T$ from Lemma 3.2 is a stripped surface glued of two strips $T_{0}$ and $T_{1}$ so that $\omega=(-1,1) \times 0$ is a leaf of type $(\mathrm{c} 2)$, see Figure 3.1 . Then Lemma 3.2 claims that these two strips can be replaced with a unique one so that the corresponding leaf of type (c2) becomes internal, i.e. having type (a).

Moreover, Corollary 3.3 shows that even infinite sequence of strips glued in a way shown in Figure 3.2 can also be replaces with one open strip.

Let $S$ be a model strip such that $\partial_{+} S=(-2,2)$ and $\partial_{-} S=(-2,2)$, see Figure 3.3.

Define the following two homeomorphisms $\phi_{c}, \phi_{m}: \partial_{+} S \rightarrow \partial_{-} S$ by

$$
\phi_{c}(t)=t, \quad \phi_{m}(t)=-t,
$$




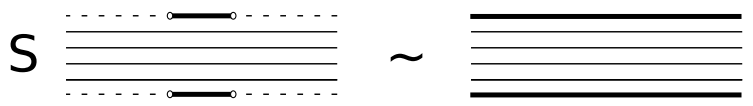

Fig. 3.3

for all $t \in(-2,2)$. Let also $C=S / \phi_{c}, M=S / \phi_{m}$ be the quotients of $S$ obtained by identifying its boundary components via $\phi_{c}$ and $\phi_{m}$ respectively. It follows from Lemma 3.2 that $C$ is an open cylinder and $M$ is an open Möbius band. Moreover, the leaf obtained by gluing $\partial_{+} S$ with $\partial_{-} S$ is of type (c1).

Lemma 3.5 If $Z$ has a leaf of type (c1), then it is $\mathcal{F}$-homeomorphic either with $C$ or with $M$.

Proof Suppose $\omega=\partial_{-} \widehat{S}_{\gamma}=\partial_{+} \widehat{S}_{\gamma}$ is a leaf of type (c1) for some $\gamma \in \mathbf{A}$. Let also $(a, b) \times\{-1\}=\partial_{-} S_{\gamma}$ and $(c, d) \times\{1\}=\partial_{+} S_{\gamma}$ be the boundary components of $S_{\gamma}$, so

$$
S_{\gamma}=(a, b) \times\{-1\} \bigcup \mathbb{R} \times(-1,1) \bigcup(c, d) \times\{1\} .
$$

Since $\omega=\partial_{-} \widehat{S}_{\gamma}=\partial_{+} \widehat{S}_{\gamma}$, it follows that

$$
S_{\gamma}=q^{-1}\left(\widehat{S}_{\gamma}\right) \subset \bigsqcup_{\alpha \in \mathbf{A}} S_{\alpha} .
$$

But $S_{\gamma}$ is an open closed subset of $\bigsqcup_{\alpha \in \mathbf{A}} S_{\alpha}$, whence from the definition of factor topology on $Z$ it follows that $\widehat{S}_{\gamma}$ is open closed in $Z$, and so it coincides with $Z$.

Thus $Z$ is obtained by gluing $(c, d) \times\{+1\}$ with $(a, b) \times\{-1\}$ by an affine homeomorphism $\phi_{\gamma}$. If $\phi_{\gamma}$ preserves orientation then $Z$ is homeomorphic with $C$. Otherwise, $Z$ is homeomorphic with $M$.

Definition 3.6 A stripped surface will be called reduced if it has no leaves of types (c1) and (c2) that is every leaf of type (c) is of type (c3).

Theorem 3.7 Every connected stripped surface $Z$ with countable base is $\mathcal{F}$ homeomorphic either to a cylinder $C$ or to a Möbius band $M$, or to a reduced surface.

Proof Since $Z$ has countable base, it follows that the number of strips in $Z$ is at most countable.

We will now define a certain graph $G$ in the following way:

- the vertices of $G$ are strips of $Z$ containing leaves of types (c1) or (c2);

- if there exists a leaf $\omega$ of type (c1) such that $\omega=\partial_{-} \widehat{S}_{\gamma}=\partial_{+} \widehat{S}_{\gamma}$ for some $\gamma \in \mathbf{A}$, then we assume that $\omega$ is a loop at vertex $\widehat{S}_{\gamma}$; 
- two such vertices $\widehat{S}_{\gamma}$ and $\widehat{S}_{\gamma^{\prime}}$ are connected by an edge in $G$ if and only if there exists a leaf $\omega$ of type (c2) such that $\omega=\partial_{-} \widehat{S}_{\gamma}=\partial_{+} \widehat{S}_{\gamma^{\prime}}$ or $\omega=\partial_{+} \widehat{S}_{\gamma}=$ $\partial_{-} \widehat{S}_{\gamma^{\prime}}$.

It follows that each vertex of $G$ has degree either 1 or 2 . Therefore every connected component of $G$ has one of the forms (i)-(v) shown in Figure 3.4.

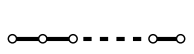

(i) finite path

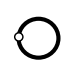

(ii) loop

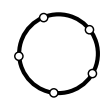

(iii) cycle (iv) infinite path in one direction (v) infinite path in both directions

Fig. 3.4

Suppose $G$ is non-empty and let $K$ be a connected component of $G$. Consider the cases (i)-(v) of Figure 3.4.

(i) Suppose $K$ is a finite non-closed path consisting of $n$ edges. As noted in Remark 3.4 each leaf of type (c2) can be regarded as an internal one after changing partition of $Z$ by strips without changing foliation $\mathcal{F}_{Z}$. This means that each individual edge of $G$ with distinct ends can be reduced. Applying Lemma $3.2 n$ times one can completely reduce all edges of $K$.

(ii) Suppose $K$ is a loop, so its edge corresponds to a leaf of type (c1). Then by Lemma $3.5 Z$ is $\mathcal{F}$-homeomorphic either with $C$ or with $M$.

(iii) Suppose $K$ is a cycle of $n$ edges. Then by arguments similar to (i) the situation reduces to the case (ii).

(iv) Suppose $K$ is an infinite in one direction path. Then the arguments of the case (i) can not be applied as it requires to consider infinite sequence.

Let $\widehat{S}_{0}, \widehat{S}_{1}, \ldots$ be an infinite sequence of strips in $Z$ corresponding to vertices of $K$ such that $\omega_{i}, i \geq 0$ is a leaf of type (c2) being an edge between vertices $\widehat{S}_{i}$ and $\widehat{S}_{i+1}$ in $K$. Interchanging $\partial_{-} S_{i}$ with $\partial_{+} S_{i}$ one may assume that

$$
\omega_{i}=\partial_{+} \widehat{S}_{i}=\partial_{-} \widehat{S}_{i+1}, \quad i \geq 0 .
$$

Notice that each $S_{i}$ is embedded into $Z$. Denote $T=\cup_{i=1}^{\infty} \widehat{S}_{i}$, so $\widehat{S}_{0}$ is omitted. Then it follows from Corollary 3.3 that $T$ is $\mathcal{F}$-homeomorphic to a model strip having only one boundary component.

Lemma 3.7.1 $\omega_{0}$ splits $Z$ so that $T \backslash \omega_{0}$ is a connected component of $Z \backslash \omega_{0}$. 
Proof It suffices to show that $T \backslash \omega_{0}$ is open and closed in $Z \backslash \omega_{0}$.

1) First we will check the openness of $T \backslash \omega_{0}$. Let $x \in T \backslash \omega_{0}$.

a) If $x \in \operatorname{Int} \widehat{S}_{i} \subset T$ for some $i \geq 1$, then $\operatorname{Int} \widehat{S}_{i}$ is an open neighborhood of $x$ in $T$.

b) If $x \in \omega_{i}=\partial_{+} \widehat{S}_{i}=\partial_{-} \widehat{S}_{i+1}, i \geq 1$, then $x$ has an open neighborhood $U$ intersecting $S_{i}$ and $S_{i+1}$ only, and so $U \subset T$.

Thus $T \backslash \omega_{0}$ is open in $Z \backslash \omega_{0}$.

2) Now let us show that $T \backslash \omega_{0}$ is closed in $Z \backslash \omega_{0}$. Let $\left\{x_{j}\right\}_{j \in \mathbb{N}} \subset T \backslash \omega_{0}$ be a sequence converging to some $x \in Z \backslash \omega_{0}$. We should prove that $x \in T \backslash \omega_{0}$. Consider two cases.

a) Suppose $x \in \operatorname{Int} \widehat{S}_{\alpha}$ for some $\alpha \in \mathbf{A}$. Since $\operatorname{Int} \widehat{S}_{\alpha}$ is an open neighborhood of $x$ in $Z \backslash \omega_{0}$, it follows that $x_{j} \in \operatorname{Int} \widehat{S}_{\alpha}$ for some $j \in \mathbb{N}$. But $x_{j} \in \widehat{S}_{i}$ for some $i \geq 1$, whence $x \in \widehat{S}_{\alpha}=\widehat{S}_{i} \subset T$ as well.

b) Suppose $x \in \partial_{-} \widehat{S}_{\alpha} \cap \partial_{+} \widehat{S}_{\alpha^{\prime}}$ for some $\alpha, \alpha^{\prime} \in \mathbf{A}$. Then $x$ has a neighborhood $U$ intersecting $\widehat{S}_{\alpha}$ and $\widehat{S}_{\alpha^{\prime}}$ only. Take $x_{j} \in U$. Then $x_{j} \in \widehat{S}_{\alpha} \cup \widehat{S}_{\alpha^{\prime}}$ which implies that one of these strips coincides with $\widehat{S}_{i}$ for some $i \geq 1$. But $\partial \widehat{S}_{i}$ intersects only $\partial \widehat{S}_{i+1}$ and $\partial \widehat{S}_{i-1}$ for $i>1$. Hence $\widehat{S}_{\alpha}$ and $\widehat{S}_{\alpha^{\prime}}$ are contained in $T$, and so $x \in T$ as well.

It follows from this lemma that one can replace $T$ with a model strip, and so the situation reduces to the case when $K$ consists of a unique edge with distinct vertices. Then by the case (i) it can be completely eliminated.

(v) Finally suppose that $K$ is an infinite in two directions path. By arguments of (iv) one can replace each of infinite ends of $K$ with a model strip, and then by (i) eliminate all edges of $K$. This also implies that $Z$ is $\mathcal{F}$-homeomorphic with an open model strip $\mathbb{R} \times(-1,1)$.

Since each strip $\widehat{S}_{\alpha}$ may correspond to at most one connected component of $G$, it follows that one can apply cases (i)-(v) mutually to each of the connected components of $G$. This allows to eliminate all leaves of type (c2) or prove that $Z$ is $\mathcal{F}$-homeomorphic either with $C$ or with $M$.

\section{Homeomorphisms group of $\mathcal{F}_{Z}$}

Let $Z$ be a stripped surface. Denote by $\mathcal{H}\left(\mathcal{F}_{Z}\right)$ the group of $\mathcal{F}$-homeomorphisms of $Z$ preserving $\mathcal{F}_{Z}$, that is for each $h \in \mathcal{H}\left(\mathcal{F}_{Z}\right)$ and each leaf $\omega \in \mathcal{F}_{Z}$ the image $h(\omega)$ is a leaf of $\mathcal{F}_{Z}$ as well.

Let also $\mathcal{H}_{0}\left(\mathcal{F}_{Z}\right)^{\prime}$ be the subgroup of $\mathcal{H}\left(\mathcal{F}_{Z}\right)$ consisting of homeomorphisms $h$ such that $h(\omega)=\omega$ for each leaf of $\mathcal{F}_{Z}$ and $h$ preserves orientation of $\omega$. 
Endow $\mathcal{H}\left(\mathcal{F}_{Z}\right)$ with the corresponding compact open topology and let $\mathcal{H}_{0}\left(\mathcal{F}_{Z}\right)$ be the identity path component of $\mathcal{H}\left(\mathcal{F}_{Z}\right)$, so it consists of homeomorphisms $h \in \mathcal{H}\left(\mathcal{F}_{Z}\right)$ isotopic in $\mathcal{H}\left(\mathcal{F}_{Z}\right)$ to id $Z$.

Let $\Sigma\left(\mathcal{F}_{Z}\right)$ be the union of leaves of types (b) and (c3). Evidently, $h\left(\Sigma\left(\mathcal{F}_{Z}\right)\right)=\Sigma\left(\mathcal{F}_{Z}\right)$ for each $h \in \mathcal{H}\left(\mathcal{F}_{Z}\right)$.

First we will consider the case when $Z$ is a model strip.

Lemma 4.1 Let $S \subset \mathbb{R} \times[-1,1]$ be a model strip and $g \in \mathcal{H}\left(\mathcal{F}_{S}\right)$. Then

$$
g(x, y)=(\lambda(x, y), \mu(y))
$$

where $\mu:[-1,1] \rightarrow[-1,1]$ is a homeomorphism, and $\lambda: S \rightarrow \mathbb{R}$ is a continuous function such that for each $y \in(-1,1)$ the correspondence $x \mapsto \lambda(x, y)$ is a homeomorphism $\mathbb{R} \rightarrow \mathbb{R}$.

Proof Since $g$ preserves leaves of $\mathcal{F}_{S}$, i.e. the lines $\mathbb{R} \times y, y \in(-1,1)$, it follows that $\mu$ does not depend on $x$. Moreover, as $g$ homeomorphically maps leaves onto leaves, the map $x \mapsto \lambda(x, y)$ is a homeomorphism $\mathbb{R} \times y \rightarrow \mathbb{R} \times \mu(y)$. Finally, $\mu:(-1,1) \rightarrow(-1,1)$ is a strictly monotone surjective continuous function, for each $y \in(-1,1)$. Therefore it extends to a self-homeomorphism of $[-1,1]$.

The following lemma is easy and we leave it for the reader.

Lemma 4.2 Let $\widehat{S} \subset Z$ be a strip containing leaves from $\Sigma\left(\mathcal{F}_{Z}\right), \xi: S \rightarrow \widehat{S}$ be the corresponding chart, and $h \in \mathcal{H}\left(\mathcal{F}_{Z}\right)$. If $h(\widehat{S})=\widehat{S}$, then $h$ lifts to a homeomorphism $g: S \rightarrow S$ of the model strip $S$ such that $\xi \circ g=h \circ \xi$.

Lemma 4.3 The group $\mathcal{H}_{0}\left(\mathcal{F}_{Z}\right)^{\prime}$ is contractible.

Proof Let $\xi: S \rightarrow \widehat{S}$ be a strip of $Z$ and $h \in \mathcal{H}_{0}\left(\mathcal{F}_{Z}\right)^{\prime}$. By assumption $h(\widehat{S})=\widehat{S}$, whence by Lemma $\left.4.2 h\right|_{\widehat{S}}$ lifts to a self-homeomorphism $g: S \rightarrow S$ such that $\xi \circ g=h \circ \xi$. Moreover, by Lemma 4.1 and assumption that $h$ preserves leaves with their orientations we have that

$$
g(x, y)=(\lambda(x, y), y)
$$

where the correspondence $x \mapsto \lambda(x, y), y \in[-1,1]$, is a self homeomorphism of $\mathbb{R}$ preserving orientation.

Then an isotopy $G: S \times[0,1] \rightarrow S$ between $g$ and $\operatorname{id}_{S}$ can be defined by the formula:

$$
G(x, y ; t)=((1-t) \lambda(x, y)+t x, y)
$$

for $(x, y) \in S \subset \mathbb{R} \times[-1,1]$. 
We will show that formulas for $G$ on distinct strips agree with affine gluing maps $\phi_{\beta}$ for all $\beta \in$ B. More precisely, let $\xi: S \rightarrow \widehat{S}$ and $\xi: S^{\prime} \rightarrow \widehat{S}^{\prime}$ be two strips of $Z$. For the convenience of notation assume that

$$
Y \subset \partial_{+} S \subset \mathbb{R} \times\{1\}, \quad X \subset \partial_{-} S^{\prime} \subset \mathbb{R} \times\{-1\},
$$

be two boundary components glued via an affine homeomorphism $\phi: Y \rightarrow X$. Let also $g: S \rightarrow S$ and $g^{\prime}: S^{\prime} \rightarrow S^{\prime}$ be the corresponding liftings of $\left.h\right|_{\widehat{S}}$ and $\left.h\right|_{\widehat{S}^{\prime}}$ respectively, and $G$ and $G^{\prime}$ the corresponding isotopies for $g$ and $g^{\prime}$ given by (4.2). We have to prove that for each $t \in[0,1]$ the following commutative diagram holds true:

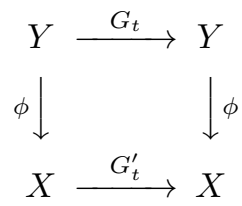

This diagram trivially holds for $t=1$ when $G_{1}$ and $G_{1}^{\prime}$ are identity maps. Moreover it also holds for $t=0$ when $G_{0}=g$ and $G_{0}^{\prime}=g^{\prime}$, since $h$ agrees on $\xi(Y)=\xi^{\prime}(X)$, so

$$
\phi \circ g=g^{\prime} \circ \phi: Y \longrightarrow X
$$

We can assume that

$$
\phi(s, 1)=(u s+v,-1), \quad g(x, 1)=(\lambda(x, 1), 1), \quad g^{\prime}(x,-1)=\left(\lambda^{\prime}(x,-1),-1\right)
$$

for some $u, v \in \mathbb{R}$, and continuous functions $\lambda: Y \rightarrow \mathbb{R}$ and $\lambda^{\prime}: X \rightarrow \mathbb{R}$. For simplicity we will also omit the second coordinate \pm 1 . Then

$$
\phi(s)=u s+v,\left.\quad g\right|_{Y}(x)=\lambda(x),\left.\quad g^{\prime}\right|_{X}(x)=\lambda^{\prime}(x),
$$

and so we get from (4.3) that

$$
\left.\phi \circ g\right|_{Y}(x)=u \lambda(x)+v=\lambda^{\prime}(u x+v)=\left.g^{\prime}\right|_{X} \circ \phi .
$$

Hence

$$
\begin{aligned}
\phi \circ G_{t}(x) & =u((1-t) \lambda(x)+t x)+v \\
& =(1-t)(u \lambda(x)+v)+t(u x+v) \\
& =(1-t) \lambda^{\prime}(u x+v)+t(u x+v) \\
& =G_{t}^{\prime} \circ \phi(x) .
\end{aligned}
$$

Thus isotopies (4.2) agree on distinct model strips, and therefore they yield a unique isotopy between $h$ and $\operatorname{id}_{Z}$ in $\mathcal{H}_{0}\left(\mathcal{F}_{Z}\right)^{\prime}$. One can easily check that such an isotopy is continuous in $(h, t) \in \mathcal{H}_{0}\left(\mathcal{F}_{Z}\right)^{\prime} \times I$ and so it yields a contraction of $\mathcal{H}_{0}\left(\mathcal{F}_{Z}\right)^{\prime}$. 
Theorem 4.4 Let $Z$ be a connected reduced stripped surface and $h \in \mathcal{H}\left(\mathcal{F}_{Z}\right)$. Then $h \in \mathcal{H}_{0}\left(\mathcal{F}_{Z}\right)$ if and only if the following three conditions hold:

(a) $h\left(\widehat{S}_{\alpha}\right)=\widehat{S}_{\alpha}$ for all $\alpha \in \mathbf{A}$;

(b) if $g_{\alpha}: S_{\alpha} \rightarrow S_{\alpha}, g(x, y)=(\lambda(x, y), \mu(y)), \alpha \in \mathbf{A}$, is a unique lifting of $\left.h\right|_{\widehat{S}_{\alpha}}$ given by (4.1), then $\mu$ is increasing and $\lambda(x, y)$ also increasing for each fixed $y \in(-1,1)$.

(c) h leaves invariant each leaf $\omega \subset \Sigma\left(\mathcal{F}_{Z}\right)$ and preserves its orientation.

Moreover, $\mathcal{H}_{0}\left(\mathcal{F}_{Z}\right)^{\prime}$ is a strong deformation retract of $\mathcal{H}_{0}\left(\mathcal{F}_{Z}\right)$, and in particular, $\mathcal{H}_{0}\left(\mathcal{F}_{Z}\right)$ is contractible as well.

Proof Let $Q \subset \mathcal{H}\left(\mathcal{F}_{Z}\right)$ be a subgroup consisting of maps satisfying (a), (b), (c). We should prove that $\mathcal{H}_{0}\left(\mathcal{F}_{Z}\right)=Q$.

Inclusion $\mathcal{H}_{0}\left(\mathcal{F}_{Z}\right) \subset Q$. Suppose $h \in \mathcal{H}_{0}\left(\mathcal{F}_{Z}\right)$, so there exists an isotopy $h_{t}: Z \rightarrow Z, t \in I$, such that $h_{0}=\mathrm{id}_{Z}, h_{1}=h$, and $h_{t} \in \mathcal{H}\left(\mathcal{F}_{Z}\right)$ for all $t \in I$.

(c) Since $h_{t}\left(\Sigma\left(\mathcal{F}_{Z}\right)\right)=\Sigma\left(\mathcal{F}_{Z}\right)$ and every leaf $\omega \subset \Sigma\left(\mathcal{F}_{Z}\right)$ is a path component of $\Sigma\left(\mathcal{F}_{Z}\right)$, it follows that

$$
h(\omega)=h_{1}(\omega)=h_{t}(\omega)=h_{0}(\omega)=\omega, \quad t \in I .
$$

Moreover, the restriction of $\left.h_{t}\right|_{\omega}: \omega \rightarrow \omega$ is an isotopy between $\operatorname{id}_{\omega}$ and $\left.h\right|_{\omega}$, whence $\left.h\right|_{\omega}$ preserves orientation.

(a) It follows further, that $h$ also leaves invariant every connected components of $Z \backslash \Sigma\left(\mathcal{F}_{Z}\right)$. But every such component is the interior of some strip, whence $h\left(\widehat{S}_{\alpha}\right)=\widehat{S}_{\alpha}$ for each $\alpha \in \mathbf{A}$, which completes (a).

(b) Moreover, let $\left(g_{\alpha}\right)_{t}: \operatorname{Int} S_{\alpha} \rightarrow \operatorname{Int} S_{\alpha}$ be a lifting of $\left.h_{t}\right|_{\operatorname{Int} \widehat{S}_{\alpha}}$. Then

$$
\left(g_{\alpha}\right)_{t}=\left(\lambda_{t}(x, y), \mu_{t}(y)\right)
$$

where $\lambda_{t}$ and $\mu_{t}$ are continuous in $(x, y, t)$. Moreover, $\left\{\mu_{t}\right\}_{t \in I}$ is an isotopy between $\mu_{0}=\mathrm{id}_{(-1,1)}$ and $\mu_{1}=\mu$. Hence $\mu$ is increasing.

Similarly, for each fixed $y \in(-1,1)$ the correspondence $x \mapsto \lambda_{t}(x, y)$ is also an isotopy between $\operatorname{id}_{\mathbb{R}}$ and $x \mapsto \lambda(x, y)$. Hence the latter is also an increasing function.

To prove the inverse inclusion $\mathcal{H}_{0}\left(\mathcal{F}_{Z}\right) \supset Q$ we need the following lemma:

Lemma 4.4.1 $\mathcal{H}_{0}\left(\mathcal{F}_{Z}\right)^{\prime}$ is a strong deformation retract of $Q$. 
Proof Let $h \in Q, \alpha \in \mathbf{A}$, and $g_{\alpha}: S_{\alpha} \rightarrow S_{\alpha}$ be a lifting of $h$, see Lemma 4.2. So by $(c)$

$$
g_{\alpha}(x, y)=\left(\lambda_{\alpha}(x, y), \mu_{\alpha}(y)\right)
$$

where then $\mu$ is increasing and $\lambda_{\alpha}(x, y)$ is increasing in $x$ for each fixed $y \in$ $(-1,1)$. Define an isotopy $F_{\alpha}: S_{\alpha} \times I \rightarrow S_{\alpha}$ by

$$
F_{\alpha}(x, y, t)=\left(\lambda_{\alpha}(x, y), t y+(1-t) \mu_{\alpha}(y)\right)
$$

Then $F_{\alpha}$ is fixed on $\partial S_{\alpha},\left(F_{\alpha}\right)_{0}=g_{\alpha}$ and $\left(F_{\alpha}\right)_{1}$ preserves each leaf of $\mathcal{F}_{S_{\alpha}}$ with its orientation. Hence the family of isotopies $\left\{F_{\alpha}\right\}_{\alpha \in \mathbf{A}}$ yield an isotopy $F(h): Z \times I \rightarrow I$ of $h$ to a diffeomorphism which preserves each leaf of $\mathcal{F}_{Z}$ with its orientation. In other words, $F(h)_{0}=h, F(h)_{t} \in Q$ for all $t \in I$, and $F(h)_{1} \in \mathcal{H}_{0}\left(\mathcal{F}_{Z}\right)^{\prime}$.

One can easily check that the map $F: Q \times I \rightarrow Q$ given by $F(h, t)=F(h)_{t}$ is continuous. Moreover, if $h \in \mathcal{H}_{0}\left(\mathcal{F}_{Z}\right)^{\prime}$, then in $(4.4)$ we have that $\mu_{\alpha}(y)=y$, whence $F_{\alpha}(x, y, t)=g_{\alpha}(x, y)$, and so $F(h, t)=h$ for all $t \in I$. In other words, $F$ is a deformation fixed on $\mathcal{H}_{0}\left(\mathcal{F}_{Z}\right)^{\prime}$, whence $\mathcal{H}_{0}\left(\mathcal{F}_{Z}\right)^{\prime}$ is a strong deformation retract of $Q$.

Since $\mathcal{H}_{0}\left(\mathcal{F}_{Z}\right)^{\prime}$ is connected (even contractible), it follows from this lemma that $Q$ is also connected. But $\operatorname{id}_{Z} \in Q$, whence $Q \subset \mathcal{H}_{0}\left(\mathcal{F}_{Z}\right)$. Theorem 4.4 is completed.

\section{References}

1. Stoilov S. Lectures on topological principles of the theory of analytic functions. Translated from the French by E. T. Stečkina. With a foreword by B. V . Šabat. - Izdat. "Nauka", Moscow, 1964.- P. 227.

2. Whyburn G. T. Analytic topology // Amer. Math. Soc. Colloquium Publications. 1942. - Vol. 28.

3. Kaplan Wilfred. Regular curve-families filling the plane, I // Duke Math. J. - 1940. Vol. 7. - P. 154-185.

4. Kaplan Wilfred. Regular curve-families filling the plane, II // Duke Math J. - 1941.Vol. 8. - P. 11-46.

5. Kamke E. Zur Theorie der Differentialgleichungen // Math. Ann. - 1928. - Vol. 99, no. 1. - P. 602-615. - URL: http://dx.doi.org/10.1007/BF01459115.

6. Kaplan Wilfred. Topology of level curves of harmonic functions // Trans. Amer. Math. Soc. - 1948. - Vol. 63. - P. 514-522.

7. Boothby William M. The topology of regular curve families with multiple saddle points // Amer. J. Math. - 1951. - Vol. 73. - P. 405-438.

8. Boothby William M. The topology of the level curves of harmonic functions with critical points // Amer. J. Math. - 1951. - Vol. 73. - P. 512-538.

9. Jenkins James, Morse Marston. Contour equivalent pseudoharmonic functions and pseudoconjugates // Amer. J. Math. - 1952. - Vol. 74. - P. 23-51.

10. Morse Marston. The existence of pseudoconjugates on Riemann surfaces // Fund. Math.1952. - Vol. 39. - P. 269-287 (1953). 
11. Jenkins James, Morse Marston. Conjugate nets, conformal structure, and interior transformations on open Riemann surfaces // Proc. Nat. Acad. Sci. U. S. A. - 1953. - Vol. 39. P. 1261-1268.

12. Jenkins James, Morse Marston. Curve families $F^{*}$ locally the level curves of a pseudoharmonic function // Acta Math. - 1954. - Vol. 91. - P. 1-42.

13. Jenkins James, Morse Marston. Conjugate nets on an open Riemann surface // Lectures on functions of a complex variable. - The University of Michigan Press, Ann Arbor, 1955.P. $123-185$.

14. Morse M. La construction topologique d'un réseau isotherme sur une surface ouverte // J. Math. Pures Appl. (9). - 1956. - Vol. 35. - P. 67-75.

15. Sharko V. V., Soroka Yu. Yu. Topological equivalence to a projection // Methods Funct. Anal. Topology. - 2015. - Vol. 21, no. 1. - P. 3-5.

16. Bolsinov A. V., Fomenko A. T. Vvedenie v topologiyu integriruemykh gamiltonovykh sistem (Introduction to the topology of integrable Hamiltonian systems). - Moscow : "Nauka", 1997. - P. 352. - ISBN: 5-02-013537-2.

17. Oshemkov A. A. Morse functions on two-dimensional surfaces. Coding of singularities // Trudy Mat. Inst. Steklov. - 1994. - Vol. 205, no. Novye Rezult. v Teor. Topol. Klassif. Integr. Sistem. - P. 131-140.

18. Sharko V. V. Smooth and topological equivalence of functions on surfaces // Ukraïn. Mat. Zh. - 2003. - Vol. 55, no. 5. - P. 687-700. - URL: http://dx.doi.org/10.1023/B: UKMA. $0000010259.21815 . \mathrm{d} 7$.

19. Sharko V. V. Smooth functions on non-compact surfaces // Pr. Inst. Mat. Nats. Akad. Nauk Ukr. Mat. Zastos. - 2006. - Vol. 3, no. 3. - P. 443-473.

20. Polulyakh Eugene, Yurchuk Iryna. On the pseudo-harmonic functions defined on a disk // Pr. Inst. Mat. Nats. Akad. Nauk Ukr. Mat. Zastos. - 2009. - Vol. 80. - P. 151.

21. Polulyakh Eugene. Kronrod-Reeb graphs of functions on non-compact surfaces // Ukrainian Math. Journal. - 2015. - Vol. 67, no. 3. - P. 375-396.

\section{Sergiy Maksymenko, Eugene Polulyakh}

Topology Department, Institute of Mathematics, Ukrainian National Academy of Science, Tereshchenkivska str. 3, 01601 Kyiv, Ukraine

Emails: maks@imath.kiev.ua, polulyah@imath.kiev.ua 